\title{
THE GOLDEN RATIO AND ITS APPLICATIONS IN THE DEVELOPMENT OF FUNDAMENTALS OF INTERIOR DESIGN CURRICULUM
}

\author{
Salwa Yousef ABDEL-BARI *
}

Department of Interior Design and Furniture, Faculty of Applied Arts, Helwan University, Egypt

\begin{abstract}
Relativity and proportionality in general are two of the biggest beauty secrets, and everything that surrounds us will become beautiful if its lengths are mathematically proportional and are not random lengths. The golden ratio is a ratio that has existed for thousands of years, and is found in mathematics, art and architecture. The rapid development in the field of interior design requires continuous development in the teaching courses of the Interior Design and Furniture Program, and the Fundamentals of Interior Design course was chosen because it is a foundational course aimed at developing creativity and the ability to inspire students. Where the research sheds light on some approaches to organizing the design elements according to a grid pattern derived from the construction systems in nature. Such as the golden ratio, the Fibonacci series, the Lockerbose modules, the fractal, the Frauni diagram, and it refers to the choice of the golden ratio and the Fibonacci sequence, where the first axis is exposed to their study and to the sources of their presence in nature and the applications of their use, then the research in the second axis is subjected to the study of the headquarters Fundamentals of design, and it explains its teaching methodology, networks used in teaching, and models of students' work, and the third axis deals with the developed modular network and its importance in design, and then reviews network models inspired by the golden ratio and the Fibonacci sequence, which are considered inputs developed for networks useful in enriching And the diversity of the design outputs of students.

Keywords

The Golden Ratio, Applications, Development, Fundamentals, Interior Design, Curriculum.
\end{abstract}

\section{Introduction:}

Nature is the designer's first teacher and by studying nature and analyzing the shapes of its elements, colors and structural composition. This helps to increase the outcome of visual nutrition - which is considered outside - and art draws its ideas from nature, directly and indirectly, and over different periods of time the designer tried Inspiration from the structural system, mathematical relations and aesthetic proportions in his works, which proves the designer's need for governing relationships and lineage that organize his work. These ruling relationships and lineage differ from era to era. There are many approaches to organizing the design elements according to a network pattern derived from the creation systems in nature.

\section{Research problem and its importance:}

The lack of organization of some interior designs and furniture using proportion and proportion, and also many designs inspired by the golden ratio, most of them have direct application - the application of the golden spiral shape. Mainly relying on regular modular networks only as a basis for teaching the Basics of Design course.

\footnotetext{
* Corresponding author: appliedarts@a-arts.helwan.edu.eg
} 


\section{The first axis:}

The golden ratio and its fields of application in design The ancient Greeks were among the first scientists to discover a way to express the asymmetric beauty found in the universe, such as plants, animals, insects and other natural structures. They expressed this mathematical phenomenon with the Greek letter.

phi, but today we call it the golden ratio, also known as the divine proportion, the golden mean, and the golden sectio, and this mathematical concept can be applied to the design to make it more beautiful and balanced.

It is important to know is there a difference between proportion and proportion? We can say that there is a difference and also a relationship between proportion and proportion, as the concept of proportionality has a strong relationship with the idea of proportion, but usually confuses the two ideas, the ratio expresses the numerical comparison between two things, and proportionality is the equality between two ratios. For example, the ratio is a comparison between two elements, such as the ratio of length to width, while proportionality is the relationship of equality between ratios, as shown in the following figure.

\section{What is the golden ratio?}

The golden ratio is a mathematical ratio found in nature, and when used in design it enhances natural designs

And organic that satisfies the eye aesthetically, but what exactly is the golden ratio and how can it be used to improve designs?

The golden ratio is a ratio that has existed for thousands of years, and it is present in mathematics, art and architecture, and is the ratio that we get when we divide a straight line into two unequal parts. The ratio of the largest portion to the smaller portion is equal to the ratio of the length of the whole line segment to the length of the largest segment, i.e. when we write this equation it is like this:

A: B - $(A+B)$ : A. It is a mathematical constant defined with a value of approximately 1021302 , and it is called the golden number. The golden ratio is best understood as the ratio 10618: 1 .

\section{Fibonacci sequence:}

Another form of proportions where relativity derives itself from the Fibonacci sequence, which is a natural sequence of numbers that can be found everywhere in nature, from the number of leaves on a tree to the snail shape of the shells as it can be found in famous works of art, architecture, and even in our bodies.

In the thirteenth century, the mathematician Leonardo Fibonacci tried to find a rule for knowing the multiplication of a group of rabbits, so it is said that the study of the successive rabbit 
breeding led to the discovery of Fibonacci to the sequence. The Fibonacci number depends on a simple development criterion: if we have two numbers 0 and 1, any number that follows it will be the sum of the previous two numbers. And the sequence is: $1-1-2-3-2-3-12$ and so on to infinity.

\section{The golden rectangle:}

An improved method for the number and the mathematical golden ratio, which is the golden rectangle, and it is drawn in the following steps: A square is drawn in red with a side length of 1 , then the right side of the quadrant is bisected, and this point is the center of the radius of the line connecting from the midpoint to the upper point On the left side, and an arc is drawn that cuts the line extending from the side of the square at a point, then we continue drawing the rectangle as it is in figure (6) and the golden rectangle will be blue.

\section{The golden snail:}

Using the golden helix is very similar to using the third grid rule - the design focus is in the center of the helix, using golden rectangles as dividing lines to place elements

\section{Sources of golden relativity:}

The golden ratio is everywhere, and it is not just some mathematical theories; They appear all the time in the environment around us; We find it represented in nature - which is rich in aesthetic proportions represented in the creativity of the Creator, the Great and the Most High, in humans, animals and plants and we find it represented in the arts and architecture as well. The golden ratio is characterized by beauty or preference - a sense of beauty through a sense of natural balance and visual harmony.

\section{The second axis:}

Description of the design basics course The design fundamentals course is taught at the second level for interior design and furniture students, and the deletion of the course in the first semester is: providing the student with knowledge and defining the various design fundamentals that support the design process and develop the student's ability to apply design fundamentals to interior design projects Different. By studying the primary modular networks (the orthogonal network, the oblique network, the central grid and finally the compound network, and its application to the distribution of furniture in the two-dimensional projection. As for the second semester, the aim of the course is to study the various design fundamentals that support the design process. And training to apply it to various interior design projects, by studying the method of symmetrical balance, asymmetric balance, and kinetic balance, using initial modular checks, and applying them to interior design and furniture in vertical-twodimensional sectors. 
In the beginning, we must know that the module is "standard check systems, which is the mechanism for using design systems and networks. It is also an effective tool for organizing design ideas and lines in architecture, interior design, furniture and art in general, as it is used in the design of horizontal projections." (Basics of designing vertical façades 1 course) and in vertical sectors (fundamentals of design rand decorative and structural elements.

Grids help to set the necessary areas for the functions (function), and to find proportionality and standardization of sizes, continuity of lines and the relationship of the part to the whole (figure); This facilitates the process of replication and finding fit in the design

The perpendicular network that consists of vertical and horizontal lines in which the direction of the line affects the construction or the visual formation, where the upright line shows a balance with the force of attraction, and the horizontal line gives a sense of stability, and the family network that consists of inclined lines representing an unbalanced position gives a dynamic feeling, And it is visually active.

\section{The third axis:}

Proposals to develop a design fundamentals course The research aims to develop a design fundamentals course. To develop and enrich the creativity and inspiration skill that demanded interior design and furniture, by developing the student's basic design inputs - the traditional molybdenum grid systems.

Grid systems play a big role in the success of many design work, and the use of grid systems requires skill and ability to obtain unity and balance. The network is considered one of the most successful systems used to achieve self-unity, and the network contributes to the perception and exploration of available solutions, and allows the generation of distinct dynamic designs.

\section{The developed network system:}

It is an unconventional constructive networks with a special developed mean, and it is a method or auxiliary element in the design inputs to organize the design elements, increases the richness of design ideas and provides many and varied design alternatives, as it is a group of invisible lines formulated by the student in a style or compatible engineering mathematical logic Aesthetically, the modular design in it depends on choosing a network that follows the golden ratio or the Fibonacci sequence, - the researcher's definition.

\section{The importance of an evolving grid in design:}

The design needs networks to maintain order and order to achieve continuity and unity in all parts of the design, and the importance of advanced networks is determined as follows:

- It allows the student to create and create many different logical possibilities based on a 
mathematical and engineering style.

- Linking design elements holistically.

- The transformation of the design into a balanced, structured building with aesthetic relationships and ratios.

-Save time and effort

First / networks developed using the golden ratio 101917: 1 or the golden number 101917 The developed networks depend on the use of modular units that follow the golden ratio and the modular distance is to show the grid lines in an upward direction of growth, derived from the golden spiral in nature and the golden number, and start with one unit then Its spacing is increased by 1 which is like this.

\section{Three types of cross grid layout are used, which we explain as follows:}

An orthogonal grid layout (through vertical and horizontal lines of two layers of similar shape and size, and the direction of growth is perpendicular.

- Mesh layout reflected through vertical and horizontal lines two layers similar in shape and size, with a reflection of the growth direction.

- An overlapping or overlapping grid layout (two similar in shape and size with a reversal of growth direction in addition to overlapping.

Second / developed networks using the Fibonacci sequence:

A direct grid layout, orthogonal (through vertical and horizontal lines of two layers similar in shape, size and direction of growth.

- Reflected grid layout (through vertical and horizontal lines of two layers of similar shape and size with a reflection of the direction of growth.

- An overlapping or overlapping grid layout (two similar in shape and size with a reversal of growth direction in addition to overlapping.

\section{Results:}

1- Designs that start using a mathematical logic known for aesthetic detail, balance and proportionality, their results also have balanced and proportionate aesthetic relationships and logic.

2- Developing a foundational course for the first stages of the interior design specialty leading to a major development in the following stages.

3- The developed network module is considered as one of the design input tools that facilitate obtaining various alternatives.

4- The application of the golden ratio and the Fibonacci sequence in a simulation of nature in an indirect way, which leads to an enrichment of the optical dictionary among interior 
design students, thus increasing the opportunity for creativity and innovation.

5- From analyzing the questionnaire, confirm the possibility of applying the proposed development in areas other than interior design.

\section{Discussion:}

There is a rapid development in the sciences related to design education, and the state and concerned institutions must adopt the development of courses. The research presented a proposal to develop the course on the basics of design, and the development relied on the use of the golden ratio and the Fibonacci sequence, in one type of design network, which is the orthogonal network, And a number of alternatives were obtained for this one type, and this idea can also be applied to the diagonal and central grid of all kinds, and the compound, which allows obtaining a very large number of alternatives that enrich the design ideas and increase the creative abilities of students.

\section{Recommendations:}

1- The necessity for faculty members to adopt the development of design courses to keep pace with the rapid development in design sciences.

2- The necessity of relying on a design methodology in teaching design subjects to develop thinking skills and the ability to present alternatives and diversity in outputs and to increase students' creative abilities.

3- Training students to use the networking method at the beginning of the design idea to generate a greater number of ideas.

4- Encouraging universities for faculty members to develop courses.

5- Working on creating design companies inspired by the golden ratio and the Fibonacci sequence in the diagonal, central and compound style.

\section{References}

1- https://www.companyfolders.comblog/golden-ratio-design-examples

2- https://www.canva.com/learn/what-is-the-golden-ratio/

3- Abdul Hamid, Shaima Osama: "Mathematical logic in ceramic product design, Ph.D., Faculty of Applied Arts, Helwan University, 2012.

4- Abdel-Bari, Salwa Youssef Al-Sebaei, Asma Abdel-Gawad Inspired Design Networks Inspired by Fractal Engineering for Furniture Design, "The Fourth International Conference of the College of Applied Arts (Creativity - Design - Production - Competitiveness) February 2014 - College of Applied Arts - Helwan University.

5- Maha Darwish, Yumni Muhammad Fathallah Yaqout, THE EFFECT OF ILLUSTRATIONS ON CHILD PERCEPTION THROUGH DESIGNING INFORMATION 
DISPLAY SYSTEMS IN MUSEUMS OF ALL KINDS, International Journal of Education and Learning Research, Vol. 2, No. 2, 2019, pp. 1-7.

6- Tasnim Yahya AL-SAYED, Zainab Abdel Hafiz FARGHALY, Osama Muhammad HUSSEIN, Khaled Mostafa MOHAMED, THE EFFECTIVENESS OF A KNOWLEDGE TRIP IN DEVELOPING PLANNING SKILLS FOR STUDENTS OF THE CLOTHING AND TEXTILE DEPARTMENT, International Journal of Education and Learning Research, Vol. 2, No. 2, 2019, pp. 8-15.

Received: February 7, 2020

Accepted: April 30, 2020 